\title{
Comparison between the Mammogram and Tomosynthesis for the Evaluation of Replacing FFDM Mammogram with Tomosynthesis
}

\author{
Alkandari LAA ${ }^{1 *}$ and Mohamed Frag MAA ${ }^{2}$ \\ ${ }^{1}$ Adan Hospital, Kuwait \\ ${ }^{2}$ Mobarak Alkabeer Hospital, Kuwait \\ *Corresponding author: Alkandari LAA, Senior \\ Specialist, Adan Hospital, Kuwait
}

Received: August 29, 2018; Accepted: October 10, 2018; Published: October 17, 2018

\begin{abstract}
Objective: The study has compared mammogram and tomosynthesis for the evaluation of replacing full field mammogram with tomosynthesis.

Material and Methods: The quantitative research design has been employed to assess the comparison between the mammogram and tomosynthesis. 151 patients have been selected, with the mean age of 48 years, from breast imaging unit at Mubarak Al-Kabeer Hospital. The patients with dense breast were taken for tomosynthesis.
\end{abstract}

Results: DBT and mammography have shown significant agreement, and both were found equivalent approaches in detecting nodules, masses, architectural distortion, calcification, and skin thickening (Kappa p-value $=0.000$; McNemar test $p$-value $>0.05$ ). Tomosynthesis has been found more appropriate in the detection of breast cancer. Conclusion: Digital Breast Tomosynthesis has been found more precise, and provides an imaging capability that is appropriate for the evaluation of lesion and identification of overlapping tissues.

Keywords: Breast cancer; Mammography; Tomosynthesis; Ultrasound; Digital breast tomosynthesis

\section{Introduction}

In the recent years, there have been an increase in the incidence of breast cancer globally. Breast cancer has been considered as the most common cause of death as it is diagnosed in approximately 140 out of 184 countries [1]. The development of diagnostic imaging has paved the way for the improvement and implementation of new technologies [2]. Mammogram is used to screen woman's breast, and shows the alterations along with pathologies on screen. 10 to $15 \%$ cases of breast cancer are still considered as unusable, despite the high quality of mammogram [1]. The unusable cases refer to such outputs of mammograms, which provide false negative results in regards of diagnosing breast cancer. The technique of mammography has lowered the mortality rate by $30 \%$; therefore, it is considered as an effective screening method [1]. This technique can diagnose dense breast, where overlapping tissues are obscuring abnormality.

The society of breast imaging commission is concerning such suggestions to offer guidance for the clinicians and patients on the usage of imaging to the breast cancer imaging [3]. Although, mammography has proved to be incredibly useful, but it does not provide accurate detection. The rate of breast cancer detection is likely to increase by conducting ultrasound along with mammography among women with dense breast and increased risk of developing severe complications [4]. The main advantage of the ultrasound of breast cancer is that it enhances the specificity, when used in a besieged manner.

Tomosynthesis improved the sensitivity and specificity of mammography by diagnosing the hidden malignant tumors behind the normal breast structures. The technique of breast tomosynthesis is likely to overcome the limitations, associated with the mammography technique [5]. The detection of signals on a digital detector at a specific location depends on the overall reduction of all the tissues. The recreation of data at final level of the procedure is responsible for establishing images that improve the objects from a specified height by suitable shifting of the projections relative to one another [6].

The mammography false-negative rate ranges from 8 to $66 \%$, depending on the tumor type and density of breast in symptomatic women [7]. The digital breast tomosynthesis is a $3 \mathrm{D}$ radiographic technique that gets information of $3 \mathrm{D}$ from 10 to 25 projection images as the source of $\mathrm{x}$-ray moves in a way as an arc moves above the breast1. As compared to FFDM, the overlap of tissues is reduced with the digital breast tomosynthesis because it involves series of images when the breast is imaged at different levels, instead of a single projection [8].

Traditionally, mammography has been reported better at explaining the morphology of calcifications. Calcifications are basically tiny deposits of minerals within the tissues of breast and they look like little white spots on the images, which occur due to cancer [9]. The replacement of mammography and tomosynthesis reduces the cost of false positive, false negative, and anxiety of waiting for the results. The effect of the superimposition of tissues is reduced by implementing DBT (Digital Breast Tomosynthesis) or 3D mammography as it improves the interpretations of mammographic evaluations [10].

There is an increase in the risk of breast cancer due to lack of 


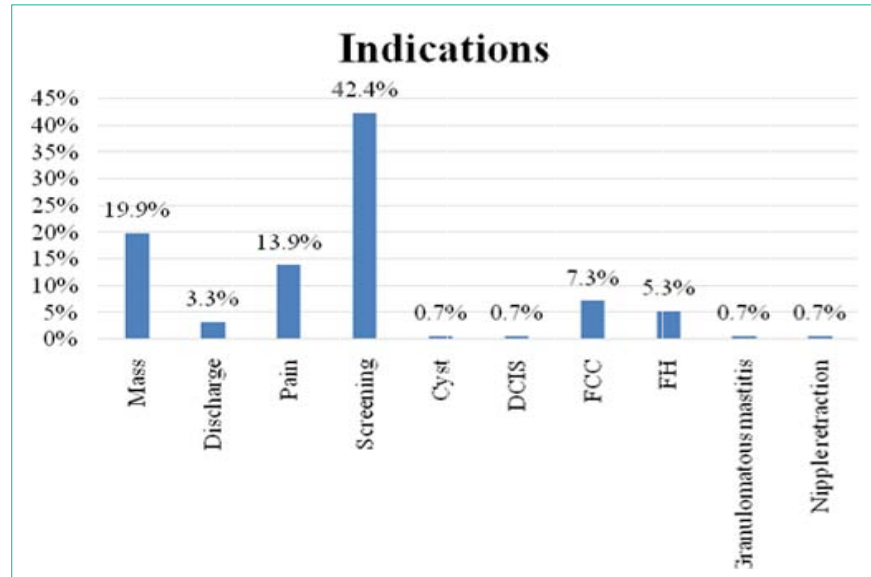

Figure 1: Indications found in the patients.

early diagnosis and proper treatment in the developing countries. In regards of mammogram, the most common limitation during screening is overlapping tissue, which obscure underlying masses and increase false negative and false positive outcomes (superimposed tissues that cause mimic cancer). As compared to the imaging techniques like magnetic resonance imaging and computerized tomography, the rate of evolution of mammographic technique has decreased. Therefore, the study has aimed to assess and compare some diagnostic cases and screening outcomes of mammogram and tomosynthesis. Moreover, the study has also evaluated the possibility of replacing tomosynthesis with Full Field Digital Mammogram (FFDM) (FFDM with tomosynthesis) without missing masses, micro calcification, and architectural distortion.

\section{Materials and Methods}

The study has incorporated quantitative research design to evaluate the comparison between the mammogram and tomosynthesis along with the possibility of replacing Full Field Digital Mammogram (FFDM) by tomosynthesis without missing masses, micro calcification, and architectural distortion. A total of 151 patients have been selected from breast imaging unit at Mubarak Al-Kabeer Hospital, a secondary care Government regional hospital. The mean age of the patients was 48 years. The referral was either from poly clinic or surgical clinics in the hospital after being inspected by the surgeon.

Complete conventional imaging was initially completed; and if the patients had dense breast, they were asked to have a tomosynthesis. If the calcifications were observed in patients, then they had additional magnification views. In few difficult cases, spot compression has also been applied additionally with the tomosynthesis. Some patients had also undergone the Digital Breast Ultrasound (DBU) if they had a mass on examination, and tomosynthesis was unable to detect it. Full Field Digital Mammography (FFDM) was performed with sono-bright (GE Health care machine) among all the patients. Any suspicious calcification worked as an indication for magnification views in lateral position.

Ultrasound was carried out with Philips US and Semines US machines, which helped in viewing craniocaudal and medio-lateral oblique. The breast was compressed in conventional manner, and the

Table 1: Indications for the Breast Cancer in women.
\begin{tabular}{|l|c|c|}
\hline Indications & N & $\%$ \\
\hline Mass & 30 & $19.90 \%$ \\
\hline Discharge & 5 & $3.30 \%$ \\
\hline Pain & 21 & $13.90 \%$ \\
\hline Screening & 64 & $42.40 \%$ \\
\hline Cyst & 1 & $0.70 \%$ \\
\hline DCIS & 1 & $0.70 \%$ \\
\hline FCC & 11 & $7.30 \%$ \\
\hline FH & 8 & $5.30 \%$ \\
\hline Granulomatous mastitis & 1 & $0.70 \%$ \\
\hline Nipple retraction & 1 & $0.70 \%$ \\
\hline
\end{tabular}
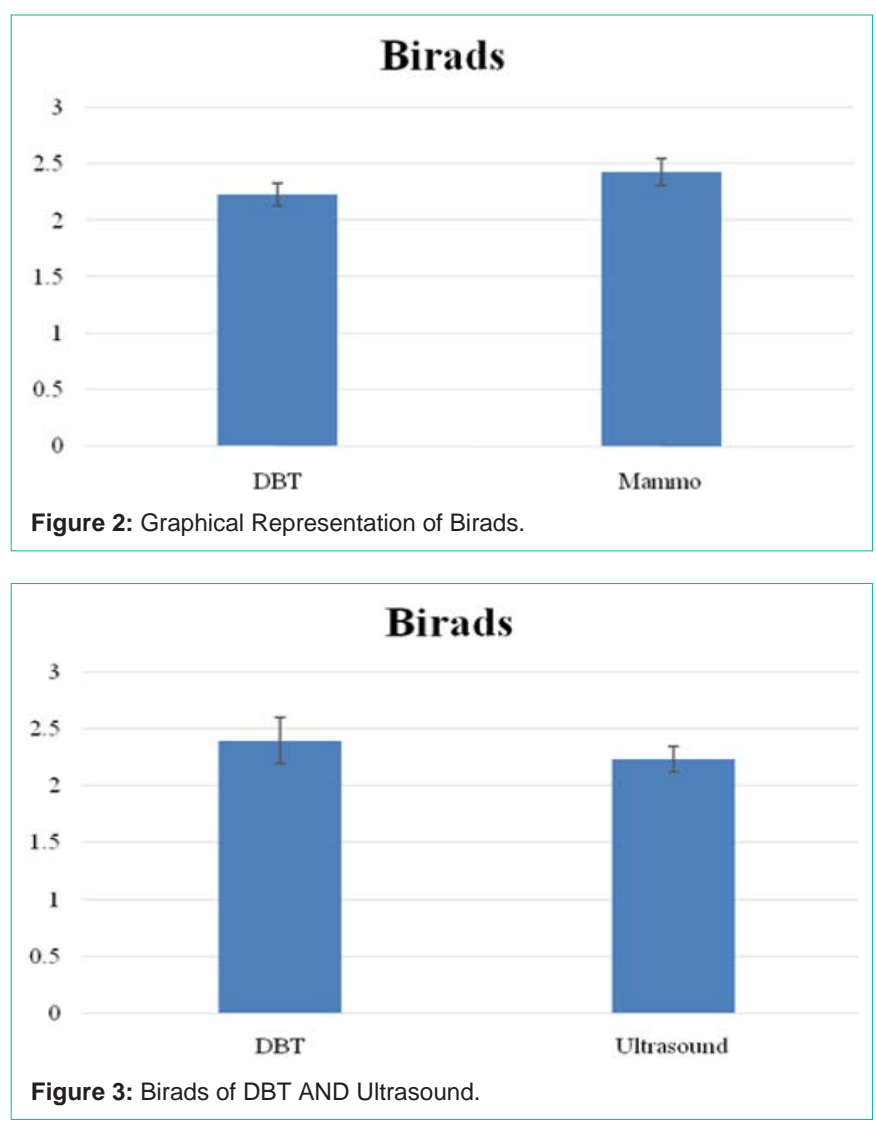

$\mathrm{X}$-ray tube moved along an arc of 15 degree. The projection images had been used to reconstruct images at $1 \mathrm{~mm}$ interval to slap image at 5 to $10 \mathrm{~mm}$ interval. The thickness of the breast had been diagnosed according to the number of the slices. The time of examination for tomosynthesis was about 15 second. The imaging interpretation and the final assessment after work up including the tomosynthesis were signed by senior radiologist. The images of the tomosynthesis were assessed in dedicated high-resolution IDI workstation (GE health care).

\section{Results}

The results of the study were based on the data, collected from 151 patients of age of mean $=48.29 \pm 8.20$ years. According to the 
Table 2: Pearson Chi Square/ Fisher's Exact Test, McNemar Test and Kappa-Value for DBT Mammography.

\begin{tabular}{|c|c|c|c|c|c|c|c|c|}
\hline Finding & & & DBT & DBT & & Pearson Chi-Square & McNemar Test & Agreement (Kappa-value) \\
\hline & & & No & Yes & Total & \multicolumn{3}{|c|}{$\mathrm{p}$-value } \\
\hline \multirow{3}{*}{ Nodules } & Mammography & No & 253 & 8 & 261 & \multirow{3}{*}{0} & \multirow{3}{*}{0.503} & \multirow{3}{*}{$0.000(0.706)$} \\
\hline & Mammography & Yes & 12 & 29 & 41 & & & \\
\hline & & Total & 265 & 37 & 302 & & & \\
\hline \multirow{3}{*}{ Mass } & Mammography & No & 225 & 20 & 245 & \multirow{3}{*}{0} & \multirow{3}{*}{0.618} & \multirow{3}{*}{$0.000(0.621)$} \\
\hline & Mammography & Yes & 16 & 41 & 57 & & & \\
\hline & & Total & 241 & 61 & 302 & & & \\
\hline \multirow{3}{*}{ Nodules/Mass } & Mammography & No & 186 & 22 & 208 & \multirow{3}{*}{0} & \multirow{3}{*}{0.878} & \multirow{3}{*}{$0.000(0.678)$} \\
\hline & Mammography & Yes & 20 & 74 & 94 & & & \\
\hline & & Total & 206 & 96 & 302 & & & \\
\hline \multirow{3}{*}{ Architectural Distortion } & Mammography & No & 291 & 1 & 292 & \multirow{3}{*}{0} & \multirow{3}{*}{0.625} & \multirow{3}{*}{$0.000(0.771)$} \\
\hline & Mammography & Yes & 3 & 7 & 10 & & & \\
\hline & & Total & 294 & 8 & 302 & & & \\
\hline \multirow{3}{*}{ Calcifications } & Mammography & No & 115 & 13 & 128 & \multirow{3}{*}{0} & \multirow{3}{*}{0} & \multirow{3}{*}{$0.000(0.595)$} \\
\hline & Mammography & Yes & 49 & 125 & 174 & & & \\
\hline & & Total & 164 & 138 & 302 & & & \\
\hline \multirow{3}{*}{ SkinThickening } & Mammography & No & 292 & 1 & 293 & \multirow{3}{*}{0} & \multirow{3}{*}{0.219} & \multirow{3}{*}{$0.000(0.562)$} \\
\hline & Mammography & Yes & 5 & 4 & 9 & & & \\
\hline & & Total & 297 & 5 & 302 & & & \\
\hline \multirow{3}{*}{ Lymph Nodes } & Mammography & No & 86 & 5 & 91 & \multirow{3}{*}{0.432} & \multirow{3}{*}{0} & \multirow{3}{*}{$0.432(0.016)$} \\
\hline & Mammography & Yes & 194 & 17 & 211 & & & \\
\hline & & Total & 280 & 22 & 302 & & & \\
\hline Assymetrical Density & & Total & 160 & 142 & 302 & 0.279 & 0 & \\
\hline
\end{tabular}

indications observed, the cases were divided into mass $(n=30)$ that is $19.9 \%$, Discharge $(n=5) 3.3 \%$, Pain $(n=21) 13.9 \%$, Screening $(n=64)$ $42.4 \%$, Cyst $(n=1) 0.7 \%$, DCIS $(n=1) 0.7 \%$, FCC $(n=11) 7.7 \%$, FH $(n=8) 5.3 \%$ (including the screening cases), granulomatous mastitis $(\mathrm{n}=1)$. $0.7 \%$, and Nipple retraction $(\mathrm{n}=1) 0.7 \%$ (Table 1$)$ and (Figure $1)$.

DBT and mammography have shown significant agreement and are equivalent in detecting nodules, masses, architectural distortion, calcification, and skin thickening (Kappa p-value $=0.000$; McNemar test $\mathrm{p}$-value $>0.05)$. The Digital Breast Tomosynthesis (DBT) and mammography have evaluated the detection of nodules as the results were statistically significant $(\alpha=0.05)$. The results of mass, architectural distortion, calcification, and skin thickening have also been observed statistically significant as the p-values observed were less than the level of significance.

The p-values observed for masses, architectural distortion, calcification, and skin thickening were 0.000 that showed their statistical significance (Table 2). DBT and mammography did not show significant agreement in detecting lymph nodes and asymmetrical densities (Kappa p-value $>0.05$; McNemar test p-value $=0.000$ ) (Table 2). DBT and mammography have not been considered equivalent tests when detecting calcifications despite of having significant agreement and association (Kappa $\mathrm{p}$-value $=0.000$; McNemar test $\mathrm{p}$-value $=0.000$ ). DBT has been identified as a better approach as compared to mammography.

Figure 2 has shown the findings after placing the values from mammogram screening for the diagnosis of breast cancer into a small number of categories. BIRADS has been used for breast screening mammography and later adopted for the use of MRI and Breast Ultrasound (US). Breasts were ranked on BIRADS scale lower on DBT as compared to mammography with a mean score of $2.23 \pm 0.85$ vs $2.43 \pm 0.95$. 47 breasts were ranked lower on DBT as compared to the mammography with a sum of 1250 ranks. 4 breasts had been ranked higher with a sum of 76 ranks. However, the reduction in BIRADS score was statistically significant (Wilcoxon Signed Ranks Test $Z=-6.114$, $p$-value $=0.000$ ).

Table 3 has shown the results of DBT and ultrasound observing small significant agreement (Kappa $=0.256, \mathrm{p}=0.000)$ and association ( $\mathrm{p}$-value $=0.000)$. However, tests were found to be in equivalent in detecting masses (McNemar test $\mathrm{p}$-value $=0.000)$. DBT and ultrasound had shown significant agreement (Kappa $=0.387, \mathrm{p}=0.000$ ), and were found equivalent in detecting skin thickening (McNemar test $\mathrm{p}$-value $=0.250)$. DBT and ultrasound did not show significant agreement $($ Kappa $=0.018, \mathrm{p}=0.555)$, or association $(\mathrm{p}$-value $=1.000)$, 
Table 3: Pearson Chi Square/ Fisher's Exact Test, McNemar Test and Kappa-Value for DBT Ultrasound.

\begin{tabular}{|c|c|c|c|c|c|c|c|c|}
\hline Finding & & & DBT & DBT & & Pearson Chi-Square & McNemar Test & Agreement (Kappa-value) \\
\hline & & & No & Yes & Total & \multicolumn{3}{|c|}{ p-value } \\
\hline \multirow{3}{*}{ Nodules/Mass } & Ultrasound & No & 184 & 64 & 248 & \multirow{3}{*}{0} & \multirow{3}{*}{0} & \multirow{3}{*}{$0.000(0.256)$} \\
\hline & Ultrasound & Yes & 22 & 32 & 54 & & & \\
\hline & & Total & 206 & 96 & 302 & & & \\
\hline \multirow{3}{*}{ SkinThickening } & Ultrasound & No & 73 & 0 & 73 & \multirow{3}{*}{0.052} & \multirow{3}{*}{0.25} & \multirow{3}{*}{$0.000(0.387)$} \\
\hline & Ultrasound & Yes & 3 & 1 & 4 & & & \\
\hline & & Total & 76 & 1 & 77 & & & \\
\hline \multirow{3}{*}{ Lymph Nodes } & Ultrasound & No & 17 & 1 & 18 & \multirow{3}{*}{1} & \multirow{3}{*}{0} & \multirow{3}{*}{$0.555(.018)$} \\
\hline & Ultrasound & Yes & 72 & 8 & 80 & & & \\
\hline & & Total & 89 & 9 & 98 & & & \\
\hline \multirow{3}{*}{ Nodules } & Ultrasound & No & 84 & 9 & 93 & \multirow{3}{*}{0.002} & \multirow{3}{*}{0.004} & \multirow{3}{*}{$0.000(.368)$} \\
\hline & Ultrasound & Yes & 0 & 3 & 3 & & & \\
\hline & & Total & 84 & 12 & 96 & & & \\
\hline \multirow{3}{*}{ Mass } & Ultrasound & No & 67 & 16 & 83 & \multirow{3}{*}{0} & \multirow{3}{*}{0.268} & \multirow{3}{*}{$0.000(.347)$} \\
\hline & Ultrasound & Yes & 24 & 27 & 51 & & & \\
\hline & & Total & 91 & 43 & 134 & & & \\
\hline
\end{tabular}

and were not equivalent in detecting lymph nodes (McNemar test $\mathrm{p}$-value $=0.000)$. Statistically significant results were found for the nodules and skin thickening as the p-values were observed less than the level of significance $(\alpha=0.05)$. Therefore, DBT has been identified as better approach as compared to ultrasound.

Figure 3 has shown the BIRADS of DBT and Ultrasound depicting the ranking of breasts on BIRAD scale on DBT similar to ultrasound with a mean score of $2.23 \pm 0.84$ vs $2.40 \pm 1.37 .25$ breasts were ranked lower on DBT as compared to ultrasound with a sum of ranks of 751. However, 24 were ranked higher with a sum of ranks 474 . The reduction in BIRADS score was statistically insignificant (Wilcoxon Signed Ranks Test $\mathrm{Z}=-1.423$, $\mathrm{p}$-value $=0.155$ ).

The combination of conventional digital mammography with the digital breast tomosynthesis decreased the screening mammography recall rates with having no negative effect on the sensitivity in the detection of malignancy [11]. Moreover, the results of Oslo trial depicted a decrease of $15 \%$ in the recall rate and increase of $27 \%$ in the detection of cancer [12]. It indicates the significance of tomography in regards of diagnosing breast cancer and showed higher rates of detecting cancers $[11,12]$.

A free response receiver operating analysis was conducted by Gur et al. [13], in which 125 tests were done and 35 cases showed the presence of cancers. The study was conducted with help of 8 radiologists, who concluded with $16 \%$ better performance with combined Full Field Digital Mammogram (FFDM); while, tomosynthesis showed 95\% CI, p <0.01. Tomosynthesis has been identified as a better approach as compared to FFDM Gur et al. [13] indicated that the CI rate of tomosynthesis was $95 \%$, which is concurrent with the results of the present study.

Noroozian [14] performed a study on 67 women having 67 masses and the results have evaluated the comparison of breast tomosynthesis to the spot views in breast masses. The study was done with 4 radiologists who were unaware to the experiments and the results concluded that performance of digital breast tomosynthesis is more helpful rather than the spot view [14]. The outcomes of study performed by Tingberg et al. [15] suggested breast tomosynthesis to be better than FFDM. The findings further elaborated that with better stage determination the accuracy in size measurement of masses is directly associated with FFDM. Cancer visibility in 36 women was compared by Anderson et al. [16]. The findings indicated that one view digital tomosynthesis and two viewed FFDM can be used collectively. 40 cancers appeared in 36 breasts, 22 of those cancers have greater visibility to digital tomosynthesis as compared to FFDM [16]. A manufacturer invented one view prototype in 2012, which has calculated high quality of $2 \mathrm{D}$ mammograms using $3 \mathrm{D}$ datasets that rendered $2 \mathrm{D}$ and $3 \mathrm{D}$ pictures from a single scan at the same time [17].

A study concluded that the need of FFDM as a part of clinical study can be eliminated by involving mammography alone or along with tomosynthesis [18]. During the DBT-based imaging, the synthetic mammography eliminates the need of additional FFDM. However, the dose-requiring FFDM in DT-based imaging can be replaced by the two-dimensional synthetic mammography [19]. The absorbed dose levels of FFDM and DBT in the clinical studies were summarized and reviewed in terms of the relative dose contribution from the Digital Breast Tomosynthesis to the Full Field Digital Mammogram. When tomosynthesis was used as a stand-alone technique, it resulted in better outcomes related to the diagnosis of masses [20].

\section{Conclusion}

Digital Breast Tomosynthesis and mammography have significant agreement and are able to detect nodules or masses successfully. The results have suggested that tomosynthesis is a modified form of mammogram and DBT that provides an imaging capability permitting more precise evaluation of lesion by allowing better delineation between the overlapping tissues. The use of this technology has a lower recall rate, positive and higher predictive value 
for the recommendation of biopsy, higher rate of cancer detection, fewer biopsies, fewer recalls, and improved confidence. The Digital Breast Tomosynthesis is valuable in the diagnostic mammography and screening mammography. Tomosynthesis has superior and comparable quality of image and has the ability to decrease the rate of recall when used adjunctively with digital screening mammography.

\section{Acknowledgement}

The authors are very thankful to all the associated personnel in any reference that contributed in/for the purpose of this research Further, this research holds no conflict of interest and is not funded through any source.

\section{References}

1. Palazuelos $\mathrm{G}$, Trujillo $\mathrm{S}$, Romero J. Breast tomosynthesis: the new age of the mammography. Rev Colomb Radiol. 2014; 25: 3926.

2. Pinsky RW, Helvie MA. Role of screening mammography in early detection/ outcome of breast cancer. New York: InDuctal carcinoma in situ and microinvasive/borderline breast cancer. 2015; 13-26.

3. Lee CH, Dershaw DD, Kopans D, Evans P, Monsees B, Monticciolo D, et al Breast cancer screening with imaging: recommendations from the Society of Breast Imaging and the ACR on the use of mammography, breast MRI, breast ultrasound, and other technologies for the detection of clinically occult breast cancer. Journal of the American college of radiology. 2010; 7: 18-27.

4. Berg WA, Blume JD, Cormack JB, Mendelson EB, Lehrer D, Bohm-Velez $M$, et al. Combined screening with ultrasound and mammography vs mammography alone in women at elevated risk of breast cancer. Jama. 2008; 299: 2151-2163.

5. Ciatto S, Houssami N, Bernardi D, Caumo F, Pellegrini M, Brunelli S, et al Integration of 3D digital mammography with tomosynthesis for population breast-cancer screening (STORM): a prospective comparison study. The lancet oncology. 2013; 14: 583-589.

6. Smith AP, Chen B, Jing Z. inventors; Hologic, Inc., assignee. Mammography/ tomosynthesis systems and methods automatically deriving breas characteristics from breast $x$-ray images and automatically adjusting image processing parameters accordingly. United States patent US. 2012; 170: 320.

7. Powell JL, Hawley JR, Lipari AM, Yildiz VO, Erdal BS, Carkaci S. Impact of the addition of Digital Breast Tomosynthesis (DBT) to standard 2D digita screening mammography on the rates of patient recall, cancer detection, and recommendations for short-term follow-up. Acad Radiol. 2017: 24: 302-307.

8. Waldherr C, Cerny P, Altermatt HJ, Berclaz G, Ciriolo M, Buser K, et al. Value of one-view breast tomosynthesis versus two-view mammography in diagnostic workup of women with clinical signs and symptoms and in women recalled from screening. AJR Am J Roentgenol. 2013; 200: 226-231.
9. Aarthy SL, Prabu S. An approach for detecting breast cancer using wavelet transforms. Indian Journal of Science and Technology. 2015.

10. Houssami N, Skaane P. Overview of the evidence on digital breas tomosynthesis in breast cancer detection. The Breast. 2013; 22: 101-108.

11. Rafferty EA, Park JM, Philpotts LE, Poplack SP, Sumkin JH, Halpern EF, et al. Assessing radiologist performance using combined digital mammography and breast tomosynthesis compared with digital mammography alone: results of a multicenter, multireader trial. Radiology. 2013; 266: 104-113.

12. Skaane P, Bandos AI, Gullien R, Eben EB, Ekseth U, Haakenaasen U, et al Comparison of digital mammography alone and digital mammography plus tomosynthesis in a population-based screening program. Radiology. 2013; 267: 47-56.

13. Gur D, Bandos Al, Rockette HE, Zuley ML, Sumkin JH, Chough DM, et al. Localized detection and classification of abnormalities on FFDM and tomosynthesis examinations rated under an FROC paradigm. AJR Am J Roentgeno. 2011; 196: 737-741.

14. Noroozian M, Hadjiiski L, Rahnama-Moghadam S, Klein KA, Jeffries DO, Pinsky RW, et al. Digital breast tomosynthesis is comparable to mammographic spot views for mass characterization. Radiology. 2012; 262: 61-68.

15. Tingberg A, Fornvik D, Mattsson S, Svahn T, Timberg P, Zackrisson S. Breast cancer screening with tomosynthesis-initial experiences. Radiation protection dosimetry. 2011; 147: 180-183.

16. Andersson I, Ikeda DM, Zackrisson S, Ruschin M, Svahn T, Timberg P, et al. Breast tomosynthesis and digital mammography: a comparison of breast cancer visibility and BIRADS classification in a population of cancers with subtle mammographic findings. Eur Radiol. 2008; 18: 2817-2825.

17. Waldherr C, Cerny P, Altermatt HJ, Berclaz G, Ciriolo M, Buser K, et al Value of one-view breast tomosynthesis versus two-view mammography in diagnostic workup of women with clinical signs and symptoms and in women recalled from screening. AJR Am J Roentgeno. 2013; 200: 226-231.

18. Zuley ML, Guo B, Catullo VJ, Chough DM, Kelly AE, Lu AH, et al. Comparison of two-dimensional synthesized mammograms versus original digital mammograms alone and in combination with tomosynthesis images. Radiology. 2014; 271: 664-671.

19. Choi JS, Han BK, Ko EY, Ko ES, Hahn SY, Shin JH, et al. Comparison between two-dimensional synthetic mammography reconstructed from digital breast tomosynthesis and full-field digital mammography for the detection of T1 breast cancer. Eur Radiol. 2016; 26: 2538-2546.

20. Svahn TM, Houssami N, Sechopoulos I, Mattsson S. Review of radiation dose estimates in digital breast tomosynthesis relative to those in two-view full-field digital mammography. The Breast. 2015; 24: 93-99.
Austin J Radiol - Volume 5 Issue 2 - 2018

ISSN : 2473-0637 | www.austinpublishinggroup.com

Alkandari et al. () All rights are reserved
Citation: Alkandari LAA and Mohamed Frag MAA. Comparison between the Mammogram and Tomosynthesis for the Evaluation of Replacing FFDM Mammogram with Tomosynthesis. Austin J Radiol. 2018; 5(2): 1082. 